\title{
Temperature of a Single Mn Atom in a CdTe Quantum Dot
}

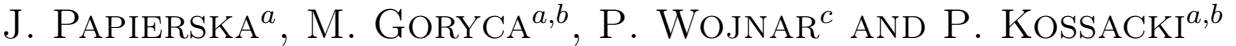 \\ ${ }^{a}$ Institute of Experimental Physics, University of Warsaw, Hoża 69, 00-681 Warsaw, Poland \\ ${ }^{b}$ Grenoble High Magnetic Field Laboratory, CNRS, Grenoble, France \\ ${ }^{c}$ Institute of Physics, Polish Academy of Sciences, Warsaw, Poland
}

\begin{abstract}
In this work we present a study of the temperature of the single magnetic atom embedded in a semiconductor quantum dot versus excitation power and magnetic field. This temperature is defined by the thermal distribution of spin states of single $\mathrm{Mn}$ ion, and results from its interaction with the neighborhood. This temperature was found to be much higher than the temperature of the thermal bath. Its dependence on the excitation power and magnetic field is discussed.
\end{abstract}

PACS numbers: 78.67.Hc, 71.70.Gm, 78.55.Et, 75.75.-c

\section{Introduction}

From the point of view of novel information storage devices a quantum dot (QD) with a single $\mathrm{Mn}$ ion is a very promising system. One of its most interesting features is the possibility of a direct read-out of the Mn spin state [1]. Furthermore, recently reported experiments exploit interaction of the Mn ion with spin-polarized excitons to realize an optical manipulation of the $\mathrm{Mn}$ spin $[2,3]$. However, to improve the storage time of the considered system, a detailed knowledge of the interactions of the Mn atom with its environment is still needed. These interactions have been studied so far by many techniques $[4,5]$.

In this work we present a study of the temperature of the single magnetic ion versus excitation power and magnetic field. This temperature is defined by the thermal distribution of spin states of single Mn ion, and results from its interaction with the neighborhood. Since it was found to be much higher than the temperature of the thermal bath, it might bring important information about $\mathrm{Mn}$ spin relaxation channels.

\section{Experimental}

Sample used in the experiment was grown by molecular beam epitaxy [6]. It contained self-assembled $\mathrm{CdTe} / \mathrm{ZnTe}$ quantum dots with very low Mn content in CdTe material so that it was possible to select a number of dots with a single Mn ion (see Fig. 1). The selection was made both spatially, by using microscope objective which allowed to excite the luminescence from the spot of about $500 \mathrm{~nm}$ in diameter, and spectrally, by selecting well separated lines from the low energy part of the spectrum.

The sample was placed in an optical cryostat and kept under constant temperature at $1.6 \mathrm{~K}$. The luminescence of the quantum dots was excited with a laser of energy larger than the band gap of the barrier material. The magnetic field, ranging from $3 \mathrm{~T}$ to $6 \mathrm{~T}$, was applied in

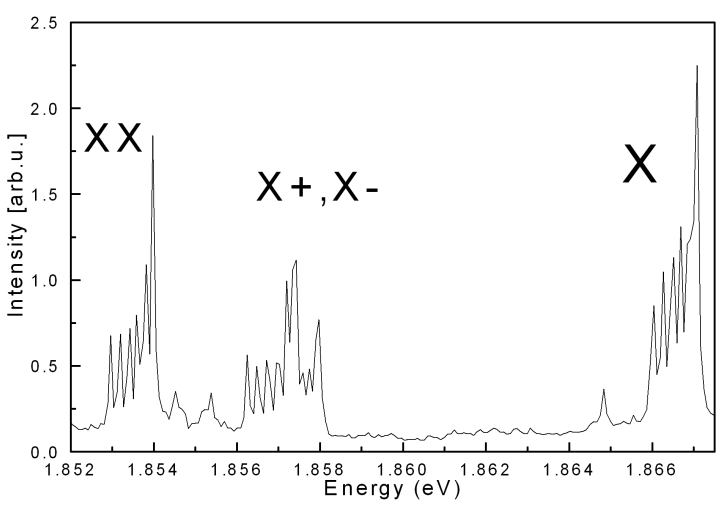

Fig. 1. An example of photoluminescence spectrum of a single quantum dot with a single $\mathrm{Mn}$ ion upon nonresonant excitation. Multiplets related to neutral (X) and charged $\left(\mathrm{X}^{+}, \mathrm{X}^{-}\right)$excitons as well as biexciton $(\mathrm{XX})$ are clearly visible.

the Faraday configuration. Spectra of the quantum dots were taken in both circular polarizations.

The temperature of the Mn ion was deduced from the relative intensities of the six excitonic lines, each corresponding to a specific Mn spin state. The intensities of these lines are well described by the Boltzmann distribution and reflect the probability of finding the magnetic atom in each spin state [1]. The temperature of the Mn atom is therefore calculated by fitting the exponential distribution to these intensities for both $\sigma^{+}$and $\sigma^{-}$polarizations.

\section{Results}

The Mn temperature was found to be high above the temperature of the thermal bath and to increase with the excitation power (see Fig. 2), reaching almost $120 \mathrm{~K}$ for $B=6 \mathrm{~T}$ and excitation power equal to $400 \mu \mathrm{W}$. The power dependence is a result of the fact that the non- 


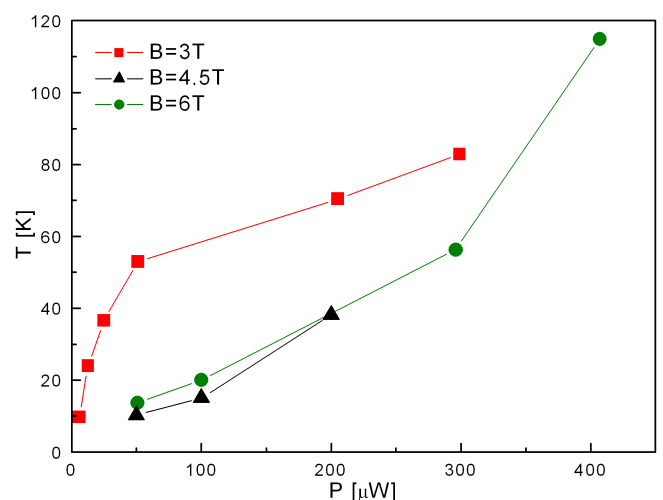

Fig. 2. Dependence of the temperature of the Mn ion on the excitation power for given values of the magnetic field.

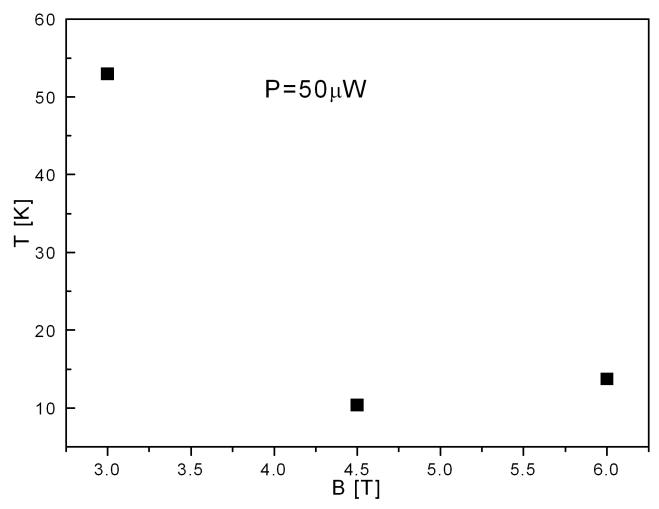

Fig. 3. Dependence of the temperature of the Mn ion on the magnetic field for excitation power equal to $50 \mu \mathrm{W}$.

-polarized photocreated excitons act as a thermal reservoir of almost infinite temperature and by increasing the excitation power we increase the relaxation channel of Mn atom with this reservoir.

The significant difference between the temperature of the $\mathrm{Mn}$ atom and the temperature of the thermal bath proves a weak coupling of the $\mathrm{Mn}$ atom to the lattice. This remains in agreement with results presented in Ref. [2], where the spin relaxation time was found to be very long, reaching $0.4 \mathrm{~ms}$ upon application of magnetic field and with no excitation of the photoluminescence.

Magnetic field clearly affects the Mn relaxation channels (see Fig. 3). For higher fields the temperature of the Mn ion decreases, which is a clear evidence of more effective spin-lattice relaxation.

One of possible origins of the decrease of Mn temperature is increase of the energy splitting between Mn spin levels which might be coupled with increased density of phonons.

\section{Conclusion}

To conclude, we performed measurements of the temperature of the single $\mathrm{Mn}$ atom in a $\mathrm{CdTe} / \mathrm{ZnTe}$ quantum dot versus excitation power and magnetic field. The Mn temperature was found to be high above the temperature of the thermal bath and to increase with the excitation power, which proves a weak coupling of the Mn atom with the lattice. The decrease of this temperature with the increasing magnetic field is probably due to increasing density of phonons of appropriate energy.

\section{Acknowledgments}

This work was partially supported by the Polish Ministry of Science and Higher Education as research grants in years 2006-2011, and by the 6th Research Framework Programme of EU (contract MTKD-CT-2005-029671).

\section{References}

[1] L. Besombes, Y. Léger, L. Maingault, D. Ferrand, H. Mariette, J. Cibert, Phys. Rev. Lett. 93, 207403 (2004).

[2] M. Goryca, T. Kazimierczuk, M. Nawrocki, A. Golnik, J.A. Gaj, P. Kossacki, P. Wojnar, G. Karczewski, Phys. Rev. Lett. 103, 087401 (2009).

[3] C. Le Gall, L. Besombes, H. Boukari, R. Kolodka, J. Cibert, H. Mariette, Phys. Rev. Lett. 102, 127402 (2009).

[4] M. Goryca, P. Kossacki, M. Nawrocki, P. Wojnar, G. Karczewski, J. Korean Phys. Soc. 53, 2963 (2008).

[5] M. Goryca, D. Ferrand, P. Kossacki, M. Nawrocki, W. Pacuski, W. Maślana, J.A. Gaj, S. Tatarenko, J. Cibert, T. Wojtowicz, G. Karczewski, Phys. Rev. Lett. 102, 046408 (2009).

[6] P. Wojnar, J. Suffczyński, K. Kowalik, A. Golnik, G. Karczewski, J. Kossut, Phys. Rev. B 75, 155301 (2007). 